\title{
RULE OPTIMIZING TECHNIQUE MOTIVATED BY HUMAN CONCEPT FORMATION
}

\author{
Fedja Hadzic and Tharam S. Dillon \\ Digital Eciossytems and Business Intelligence Institute (DEBII), Curtin Universityof Technology \\ fedja.hadzic@postgrad.curtin.edu.au,t.dillon@curtin.edu.au
}

Keywords: Data Mining, Rule Optimization, Feature Selection

Abstract: In this paper we present a rule optimizing technique motivated by the psychological studies of human concept learning. The technique allows for reasoning to happen at both higher levels of abstraction and lower level of detail in order to optimize the rule set. Information stored at the higher level allows for optimizing processes such as rule splitting. merging and deleting, while the information stored at the lower level allows for determining the attribute relevance for a particular rule.

\section{INTRODUCTION}

During the rule optimization process a trade-off usually needs to be made between the misclassification rate (MR), and coverage rate (CR) and generalization power (GP). MR corresponds to the number of incorrectly classified instances and it should be minimized. CR is the number of instances that are captured by the rule set and this should be maximized. Good GP is achieved by simplifying the rules. The trade-off occurs especially when the data set is characterized by continuous attributes where a valid constraint on the attribute range needs to be determined for a particular rule. Increasing the attribute range usually leads to the increase in CR but at the cost of an increase in MR. Similarly if the rules are too general they may lack the specificity to distinguish some domain characteristics and hence the MR would increase.

In this paper we extend the rule optimizing method presented in (Hadzic \& Dillon, 2005; Hadzic \& Dillon, 2007). The method was used to optimize the rules learned by a neural network and in this work it is extended to be applicable to rules obtained using any knowledge learning methods. The extension allows reasoning to happen at both higher level of abstraction and lower level of detail. The information about the relationships between the class attribute and the input attributes will be available for determining the relevance of rule attributes at any stage of the rule optimizing $(R O)$ process. The attributes irrelevant for a particular rule can then be deleted. Furthermore, attributes previously found as irrelevant can be re-introduced if found relevant at a later stage in the process. The proposed method is evaluated on the rules learned from publicly available real world datasets and the results indicate the effectiveness of the method.

\section{MOTIVATION}

Concept or category formation has been studied extensively in the psychology area. Generally it refers to the process by which a person learns to sort specific observations into general rules or classes. It allows one to respond to events in terms of their class membership rather than uniqueness (Bruner et al., 1956). This process is the elementary form by which humans adjust to their environment. Relevant attributes need to be identified and a rule has to be learned, developed or applied for formulating a concept (Sestito \& Dillon, 1994). Human subjects consistently seek confirming information by actively searching they environment for appropriate examples which can confirm or modify the newly discovered concepts (Kristal 1981; Pollio 1974, sestito \& Dillon 1994). Hence, there exists one level at which the concepts or categories have been formed and there is another level where the observations are used for confirming or adjusting the learned concepts and their relationships (Rosch 1977). When a formed belief appears to be contradictory for some observations one may go into thinking at the lower level of detail to investigate the constituents of that belief and what example 
observations formed it. An update of the belief can then occur whereby some pre-conditions are added or removed from the constituents of that particular belief. Re-introducing new features previously found as irrelevant or removing the irrelevant one, can occur quite frequently while learning occurs and until some reliable belief is formed.

Being able to perform this type of task is desirable for the rule optimizing process. The higher level of abstraction would correspond to the rules with the attribute constraints and the predicting class values, while at the lower level the relationships between attribute values and the occurring class values are stored. This information can be used to determine the relevance of attributes in predicting of the class value that a particular rule implies. Integrating the feature selection criterion with the rule optimizing stage is advantageous since initial bad choices made about the attribute relevance could be corrected as learning proceeds.

\section{METHOD DESCRIPTION}

The method takes as input a file describing the rules detected by a particular classifier and the domain dataset from which the rules were learned. The rules are represented in a graph structure $(G S)$ where each rule has a set of attribute constraints and points to one or more target values. The GS contains the high level information about the domain at hand in form of rules and is used for reasoning at the higher level of abstraction.

\subsection{Graph Structure Formation}

In order for the GS to be formed two files are read, one describing the rules detected by a classifier and the other containing the total set of instances from which the rules were learned. The rules are in form of attribute constraints while the implying class of each rule is ignored. The reason is that during the whole process of $R O$, the implying class values can change as some clusters will be merged or split. Rather the domain dataset is read according to which the weighted links between the rules and class values are set. The implying class value of a rule becomes the highest weighted link to a particular class value node. This class value has most frequently occurred in the instances which were captured by the rule. An example of the GS after a dataset is read in is shown in Figure 1. The implying class of Rulel and Rule 3 would be class value 1 while for Rule2 it is class value 2 . Even though it is not shown in the figure, each rule has a set of attribute constraints associated with it, which we refer to as the weight vector $(W V)$ of that rule. The set of attribute values occurring in the instance being processed are referred to as the input vector $(I V)$. Hence, to classify an instance we match the $I V$ against the $W V \mathrm{~s}$ of the available rules. A constraint for a continuous attribute is given in terms of a lower range (Ir) and an upper range (ur) indicating the set of allowed attribute values.

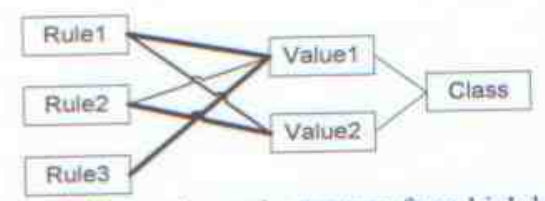

Figure 1: Example graph structure from high level

\subsection{Storing Lower Level Information}

Previous sub-section has explained the GS formation at the top level which is used mainly for determining the implying class values of the rules. In this section we discuss how lower level instance information is stored for each rule. This low level information is necessary for the reasoning at the lower level.

As previously mentioned each rule has a set of attribute constraints associated with it, which are stored in its $W V$. For each of the attributes in the $W V$ we collect the occurring attribute values in the instances that were captured by that particular rule. Hence each attribute has a value list $(V L)$ associated with it which stores all the occurring attribute values. Furthermore, each of the value objects in the list has a set of weighted links to the occurring class values in the instance where that particular value occurred. This is necessary for the feature selection process which will be explained later. For a continuous attributes there could be many occurring values and values close to one another are merged into one value object when the difference between the values is less than a chosen merge value threshold. Hence the numerical values stored in a list of a continuous attribute will be ordered so that a new value is always stored in an appropriate place and the merging can occur if necessary. Figure 2 illustrates how this low level information is stored for a rule that consists of two continuous attributes A and $\mathrm{B}$. The attribute $\mathrm{A}$ has the lower range (Ir) and the upper range (ur) in between which the values vI, $\mathrm{v} 2$ and $\mathrm{v} 3$ occur. The ' $1 \mathrm{r}$ ' of $\mathrm{A}$ is equal to the value of $\mathrm{vl}$ or the 'Ir; of $\mathrm{vl}$ if $\mathrm{vl}$ is a merged value object, while the 'ur' of $A$ is equal to the value of $v 3$ or the 'ur' of $\mathrm{v} 3$ if $\mathrm{v} 3$ is a merged value object. 


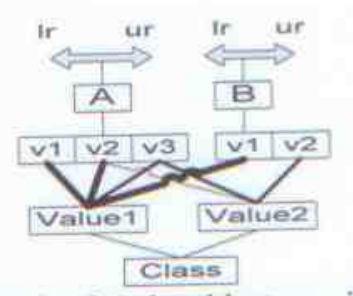

Figure 2: Storing low level instance information

\subsection{Reasoning at the Higher Level}

Once the implying classes are set for each of the rules the dataset is read in again in order to check for any misclassifications and update the rule set accordingly. When a rule captures an instance that has a different class value than the implication of the rule, a child rule will be created in order to isolate the characteristic of the rule causing the misclassification. The attribute constraints of the parent and child rule are updated so that they are exclusive from one another. The child attribute constraint ranges from the attribute value of the instance to the range limit of the parent rule to which the input attribute value was closest to. The parent rule adopts the remaining range as the constraint for the attribute at hand.

After the whole dataset is read in there could be many child rules created from a parent rule. Some child rules may be merged together first but explanation of this is to come later once we discuss the process of rule similarity comparison and merging. If a child rule points to other target values with high confidence it become a new rule and this corresponds to the process of rule splitting, since the parent rule has been modified to exclude the child rule which is now a rule on its own. On the other hand if the child rule still mainly points to the implying class value of the parent rule it is merged back into the parent rule (if they are still similar enough). An example of a rule which has been modified to contain a few children due to the misclassifications is displayed in Figure 3. The reasoning explained would merge 'Child3' back into the parent rule since it points to the implying class of the parent rule with high weight. This is assuming that they are still similar enough. On the other hand Child1 and Child2 would become new rules since they more frequently capture the instances where the class value is different to the implying class of the parent rule. Furthermore if they are similar enough they would be merged into one rule.

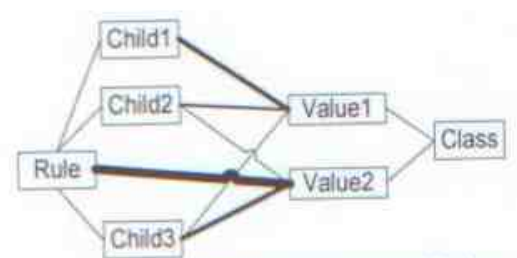

Figure 3: Example of rule splitting

In order to measure the similarity among the rules we make use of a modified Euclidean distance $(E D)$ measure. This measure is also used to determine which rule captures a presented instance. An instance is always assigned to the rule with the smallest $E D$ to the $I V$. Even though one would expect the $E D$ to be equal to 0 when classifying instances this may not always be the case throughout the $R O$ process. The $E D$ calculation is calculated according to the difference in the allowed range values of a particular attribute. The way that $E D$ is calculated is what determines the similarity among rules, and therefore we first overview the $E D$ calculation and then proceed onto explaining the merging of rules that may occur in the whole $R O$ process.

\subsubsection{ED calculation}

For a continuous attribute $a_{i}$ occurring at the position $i$ of WV of rule $R$, let ' $a_{i} l I^{\prime}$ ' denote the lower range, ' $a_{i} u r$ ' the upper range, and ' $a_{i} v$ ' the initial value if the ranges of $a_{i}$ are not set. The value from the $i-t h$ attribute of IV will be denotes as iva. The i-th term of the ED calculation between IV and WV of R for continuous attributes is:

- case 1: $a_{i}$ ranges are not set

- 0 iff $i v a_{i}=a_{i} v$

- $\quad \mathrm{iva}_{\mathrm{i}}-\mathrm{a}_{1} \mathrm{v}$ if iva; $>\mathrm{a}_{\mathrm{i}} \mathrm{v}$

- $a_{1} v-\mathrm{iva}_{i}$ if $i v a_{i}<\mathrm{a}_{4} \mathrm{v}$

- case 2: $a_{i}$ ranges are set

- $\quad 0$ iff $i v a_{i} \geq a_{i} I r$ and $i v a_{i} \leq a_{j} u r$

- $a_{i} l r-i v a_{i}$ if $i v a_{1}<a_{i} l r$

- $\quad$ iva $_{i}-a_{i} u r$ if iva $>a_{i} u r$

The input merge threshold used for continuous attribute (MT) also needs to be set with respect to the number of continuous attributes in the set. It corresponds to the maximum allowed sum of the range differences among the WV and IV so that the rule would capture the instance at hand.

When calculating the $E D$ for the purpose of merging similar rules there are four possibilities that need to be accounted with respect to the ranges being set in the rule attributes, and the $E D$ 
calculation is adjusted. For rule $R_{1}$ let $r_{1} a_{i}$ denote the attribute occurring at the position $i$ of $W V$ of rule $R_{1}$, let ' $r_{1} a_{i} \mid r$ ' denote the lower range, ' $r_{1} a_{i} u r$ ' the upper range, and ' $r_{1} a_{i} v$ ' the initial value if the ranges of $r_{1} a_{i}$ are not set. Similarly for rule $R_{2}$ let $r_{2} a_{1}$ denote the attribute occurring at the position $i$ of $W V$ of rule $R_{2}$, let ' $r_{2} a_{1} l r$ ' denote the lower range, ' $r_{2} a_{1} u r$ ' the upper range, and ' $r_{2} a_{1} v$ ' the initial value if the ranges of $r_{2} a_{4}$ are not set. The $\mathrm{j}$-th term of the $E D$ calculation between the WV of $R_{1}$ and WV of $R_{2}$ for continuous attributes is:

- case 1: both $r_{1} a_{i}$ and $r_{2} a_{1}$ ranges are not set

- 0 iff $r_{1} a_{i} v=r_{2} a_{i} v$

- $r_{1} a_{i} v-r_{2} a_{i} v$ if $r_{1} a_{3} v>r_{2} a_{1} v$

- $r_{2} a_{1} v-r_{1} a_{i} v$ if $r_{1} a_{i} v<r_{2} a_{i} v$

- case $2: r_{1} a_{i}$ ranges are set and $r_{2} a_{i}$ ranges are not set

- $\quad 0$ iff $r_{2} a_{i} v \geq r_{1} a_{1} i r$ and $r_{2} a_{i} v \leq r_{1} a_{i} u r$

- $r_{1} a_{i} \mid r-r_{2} a_{i} v$ if $r_{2} a_{i} v<r_{1} a_{i}$ ir

- $r_{2} a_{i} v-r_{1} a_{i} u r$ if $r_{2} a_{i} v>r_{1} a_{i} u r$

- case 3: $r_{1} a_{i}$ ranges are not set and $r_{2} a_{i}$ ranges are set

- 0 iff $r_{1} a_{i} v \geq r_{2} a_{i}$ ir and $r_{1} a_{i} v \leq r_{2} a_{i} u r$

- $r_{2} a_{i} i r-r_{1} a_{i} v$ if $r_{1} a_{1} v<r_{1} a_{4}, r$

- $r_{1} a_{i} v-r_{2} a_{i} u r$ if $r_{1} a_{i} v>r_{2} a_{i} u r$

- case 4: both $r_{1} a_{i}$ and $r_{2} a_{i}$ ranges are set

- 0 iff $r_{1} a_{1} l r \geq r_{2} a_{1}$ Ir and $r_{1} a_{i} u r \leq r_{2} a_{3} u r$

- 0 iff $r_{2} a_{j} l r \geq r_{1} a_{j}$ Ir and $r_{2} a_{i} u r \leq r_{1} a_{i} u r$

- $\min \left(r_{1} a_{i}\left|r-r_{2} a_{1}\right| r, r_{1} a_{i} u r-r_{2} a_{i} u r\right)$ iff $r_{1} a_{1}, r>$ $r_{2} a_{i}$ Ir and $r_{1} a_{i} u r>r_{2} a_{i} u r$

- $\min \left(r_{2} a_{i} \mid r-r_{1} a_{i} l r, r_{2} a_{i} u r-r_{1} a_{i} u r\right.$ iff $r_{2} a_{i} l r>$ $r_{1} a_{i}$ Ir and $r_{2} a_{i} u r>r_{1} a_{i} u r$

- $\quad\left(r_{1} a_{i} \mid r-r_{2} a_{j} u r\right)$ iff $r_{1} a_{i} \mid r>r_{2} a_{i} u r$

- $\quad\left(r_{2} a_{i} l r-r_{1} a_{i} u r\right)$ iff $r_{2} a_{i} l r>r_{1} a_{1} u r$

For a rule to capture an instance or for it to be considered sufficiently similar to another rule the $E D$ would need to be smaller than the $M T$ threshold.

\subsubsection{Rule merging}

As mentioned at the start of Section 3.3 the child rules may be created when a particular rule captures an instance that has a different class value than the implying class value of that rule (i.e. misclassification occurs). After the whole file is read in the child rules that have the same implying class values are merged together if the $E D$ between them is below the $M T$. Thereafter the child rules either become a new rule or are merged back into the parent rule, as discussed earlier. Once all the child rules have been validated the merging can occur among the new rule set. Hence if any of the rules have the same implying class value and the $E D$ between them is below the $M T$ the rules will be merged together and the attribute constraints updated. After this process the file is read in again and any of the rules that do not capture any instances are deleted form the rule set.

\subsection{Reasoning at the Lower Level}

Once the rules have undergone the process of splitting and merging, the relevance of rule attributes should be calculated as some attributes may have lost their relevance through merging of two or more rules. Other attributes may have become relevant as a more specific distinguishing factor of a new rule which resulted from splitting of an original rule. For this purpose we make use of the symmetrical tau (Zhou \& Dillon, 1991) feature selection criterion whose calculation is made possible by the information stored at the lower level of the graph structure. We start this section by discussing the properties of the symmetrical tau and then proceed onto explaining how the relevance cut-off is determined and the issue of choosing the merge value threshold for the value objects in a value list.

\subsubsection{Feature Selection Criterion}

Symmetrical Tau (c) (Zhou \& Dillon, 1991) is a statistical measure for the capability of an attribute in predicting the class of another attribute. The $\tau$ measure is calculated using a contingency table which is used in statistical area to record and analyze the relationship between two or more variables. If there are $\mathrm{I}$ rows and $\mathrm{J}$ columns in the table, the probability that an individual belongs to row category $i$ and column category $j$ is represented as $P(i j)$, and $P(i+)$ and $P(+j)$ are the marginal probabilities in row category $i$ and column category $j$ respectively, the Symmetrical Tau measure is defined as (Zhou \& Dillon, 1991):

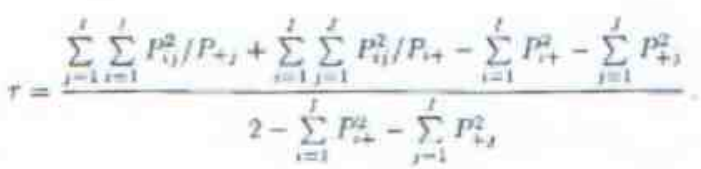

For the purpose of feature selection problem one criteria in the contingency table could be viewed as an attribute and the other as the target class that needs to be predicted. In our case the information contained in a contingency table between the rule attributes and the class attributes is stored at the lower level of the graph structure as explained in Section 3.2. The $\tau$ measure was used as a filter approach for the feature subset selection problem in 
(Hadzic \& Dillon, 2006). In the current work its capability of measuring the sequential variation of an attribute's predictive capability is exploited.

\subsubsection{Determining Relevance Cut-off}

For each of the rules that are triggered for multiple class values we calculate the $\tau$ criterion and rank the rule attributes according to the decreasing $\tau$ value. The relevance cut-off point is determined as the point in the ranking where the $\tau$ value of an attribute is less than half of the previous attribute's $\tau$ value. All the attributes below the cut-off point are considered irrelevant for that particular rule and are removed from the rule's WV. On the other hand, if some of the attributes above the relevance cut-off point were previously excluded from the WV of the rule, they are now re-introduced since their $\tau$ value indicates their relevance for the rule at hand.

As mentioned in Section 3.2 when the occurring values stored in the value list of an attribute are close together they are merged and the new value object represents a range of values. The merge value threshold chosen determines when the difference among the value objects is sufficiently small for merging to occur. This is important for appropriate $\tau$ calculation. Ideally a good merge value threshold will be picked with respect to the value distribution of that particular attribute. However, this information is not always available and in our approach we pick a general merge threshold of around 0.02 . This has some implications for the calculated $\tau$ value since when the categories of an attribute $A$ are increased more is known about attribute $A$ and the error in predicting attribute B may decrease. Hence, if the merge value threshold is too large many attributes will be considered as irrelevant since all the occurring values could be merged into one value object which points to many target objects and this aspect would indicate no distinguishing property of the attribute. On the other hand, if it is too small many value objects may exist which may wrongly indicate that the attribute has high relevance in predicting the class attribute.

\section{METHOD EVALUATION}

The proposed method was evaluated on two rule sets learned from publicly available real world datasets (Blake et al., 1998). The rule optimizing process was run for 10 iterations for each of the tested domains.

The first set of rules we consider has been learned from the 'Iris' dataset using the continuous self-organizing map (Hadzic \& Dillon, 2005) so that we can compare the improvement of the extension to the rule optimizing method. The merge cluster threshold $M T$ was set to 0.1 and the merge value threshold $M V T$ for attribute values was set to 0.02 . The rules obtained using the CSOM technique (Hadzic \& Dillon, 2005) are displayed in Figure 4. When the rules obtained after retraining were taken as input by our proposed rule optimization method the resulting rule set was different in only one rule. The rule 4 was further simplified to exclude the attribute constraint from sepal-width and the new attribute constraint was only that petal-width has to be between the values of 0.667 and 1.0 for the class value of Iris-virginica. Hence the process was able to detect another attribute that has become irrelevant during the $R O$ process. The predictive accuracy remained the same.

\begin{tabular}{|c|c|c|c|c|c|c|c|}
\hline Q & Canctraint & Inglierbr & $\mathrm{IR}$ & $\theta$ & Cantrint & Iungflech: & XIR \\
\hline$T$ & 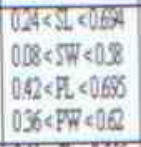 & $\begin{array}{l}15-2 \\
1 \pi-1\end{array}$ & 7 & 1 & 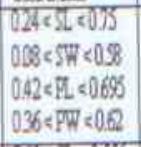 & $\begin{array}{l}\ln -11 \\
\ln -1\end{array}$ & 1 \\
\hline 2 & 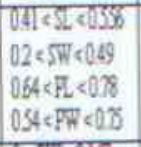 & $1 \pi-9$ & & 2 & 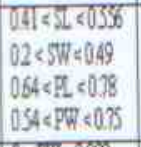 & Iro-1 & \\
\hline 3 & DePWदण & $\mathrm{b}-33$ & & 3 & DSPW CODB & $5-15$ & \\
\hline 4 & 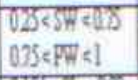 & $78-21$ & & 4 & $\begin{array}{l}02 \mathrm{cSW}=073 \\
0.660 \mathrm{PW}<1\end{array}$ & $16-12$ & \\
\hline 3 & 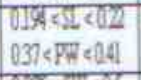 & $1 \pi-2$ & & 3 & $\begin{array}{l}019 \times 5[2122 \\
037<\mathrm{PW}<041\end{array}$ & $10 x-1$ & \\
\hline 6 & $\begin{array}{l}030 \times 34<05 \\
0625 \leqslant P W<07\end{array}$ & $\begin{array}{l}\sqrt{\mathrm{g}-5} \\
18-1\end{array}$ & $T$ & 6 & $\begin{array}{l}035<5 \times 005 \\
068<P W \div 0 . ?\end{array}$ & In-1 & \\
\hline
\end{tabular}

Figure 4: Iris rule set as obtained by using the traditional rule optimizing technique

With respect to using $\mathrm{CSOM}$ to extract rules from the 'Iris' domain we have performed another experiment. The initial rules extracted by CSOM without the network pruning and retraining of the network were optimized. When network pruning occurs the network should be re-trained for new abstractions to be properly formed. In this experiment we wanted to see how the $R O$ technique performs by itself without any network pruning or retraining.

\begin{tabular}{|l|l|}
\hline Rules & Implying class \\
\hline $0.33<\mathrm{PL}<0.678$ & Iris-versicolor \\
$0.375<\mathrm{PW}<0.792$ & \\
\hline $0.208<\mathrm{SW}<0.542$ & Iris-virginica \\
$0.627<\mathrm{PL}<0.847$ & \\
$0.54<\mathrm{PW}<1.0$ & \\
\hline $0.778<\mathrm{SL}<1.0$ & Iris-virginica \\
$0.25<\mathrm{SW}<0.75$ & \\
\hline
\end{tabular}




\begin{tabular}{|l|l|}
\hline $0.814<\mathrm{PL}<1.0$ & \\
$0.625<\mathrm{PW}<0.917$ & \\
\hline $0.0<\mathrm{SL}<0.417$ & Iris-setosa \\
$0.41<\mathrm{SW}<0.917$ & \\
$0.0<\mathrm{PL}<0.153$ & \\
$0.0<\mathrm{PW}<0.208$ & \\
\hline
\end{tabular}

Figure 5. Optimized initial rules extracted by CSOM Notation: SL - sepal_length, SW - sepal_width, PL petal_length, PW-petal_width

By applying the $R O$ technique the rule set was reduced to four rules as displayed in Figure 5. However, not as many attributes were removed from each of the rules and two instances were misclassified. Hence, performing network pruning and retraining prior to $R O$ may achieve a more optimal rule set. However, in the cases where retraining the network may be too expensive the $R O$ technique can be applied by itself. In fact compared to the initial set of rules detected by CSOM, which consisted of nine rules with three misclassified instances this is still a significant improvement.

The second set of experiments was performed on the complex 'Sonar' dataset which consists of sixty continuous attributes. The examples are classified into two groups one identified as rocks $(R)$ and the second identified as metal cylinders (M). The learned decision tree by the C4 algorithm (Quinlan, 1990) consisted of 18 rules with the predictive accuracy equal to $65.1 \%$. These rules were taken as input in our $R O$ technique and the $M T$ was set to 0.2 while the $M V T$ was set to 0.0005 . The optimized rule set consisted of only two rules i.e $0.0<$ all $<=0.197$ $\rightarrow \mathrm{R}$ and $0.197<\mathrm{all}<=1.0 \rightarrow \mathrm{M}$. When tested on an unseen dataset the predictive accuracy was 82.2 $\%$ i.e. 11 instances were misclassified from the available 62 . Hence the $R O$ process has again proved useful in simplifying the rules set without the cost of increasing the number of misclassified instances.

\section{CONCLUSIONS}

This paper has presented a rule optimizing technique motivated by the psychological studies of human concept information. The capability to swap from the higher level reasoning to the reasoning at the lower instance level has indeed proven useful for determining the relevance of attributes throughout the rule optimizing process. The method is applicable to the optimization of rules obtained from any data mining techniques. The evaluation of the method on the rules learned from real world data by different classifier methods has shown its effectiveness in optimizing the rule set. As a future work method needs to be extended so that categorical attributes can be handled as well. Furthermore, it would be interesting to explore the possibilities of the rule optimizing method in becoming a stand-alone machine learning method itself.

\section{REFERENCES}

Blake, C., Keogh, E. and Merz, C.J., 1998. UCI Repository of Machine Learning Databases, Irvine, CA: University of California, Department of Information and Computer Science, 1998. [http://www.ics.uci.edu/ -mlearn/MLRepository.html].

Bruner, J.S., Goodnow, J.J., and Austin, G.A., 2001. A study of thinking, John Wiley \& Sons, Inc., New York. 1956.

Hadzic, F. \& Dillon, T.S., 2005. “CSOM: Self Organizing Map for Continuous Data", $3^{\text {nd }}$ Int 'l IEEE Conf. on Industrial Informatics, 10-12 August, Perth.

Hadzic, F, and Dillon, T.S., 2006 "Using the Symmetrical Tau $(\tau$ ) Criterion for Feature Selection in Decision Tree and Neural Network Learning", $2^{\text {nd }}$ Workshop on Feature Selection for Data Mining: Interfacing Machine Learning and Statistics, in conj. with SIAM Int'l Conf. on Data Mining, Bethesda, 2006.

Hadzic, F. \& Dillon, T.S., 2007. "CSOM for Mixed Data Types", $4^{\text {th }}$ Int'l Symposium on Neural Networks, June 3-7, Nanjing, China.

Kristal, L., ed. 1981, ABC of Psychology, Michael Joseph, London, pp. 56-57.

Pollio, H.R., 1974, The psychology of Symbolic Activity, Addison-Wesley, Reading, Massachusetts.

Quinlan, J.R., 1990. "Probabilistic Decision Trees". Machine Learning: An Artificial Intelligence Approach Volume 4, Kadratoff, Y \& Michalski, R. Morgan Kaufmann Publishers, Inc., San Mateo, California.

Roch, E. 1977, "Classification of real-world objects: Origins and representations in cognition", in Thinking: Readings in Cognitive Science, eds P.N. JohnsonLaird \& P.C. Wason, Cambridge University Press, Cambridge, pp. 212-222.

Sestito, S. and Dillon, S.T., 1994. Automated Knowledge Acquisition. Prentice Hall of Australia Pty Ltd, Sydney.

Zhou, X., and Dillon, T.S., 1991. "A statistical-heuristic feature selection criterion for decision tree induction". IEEE Transactions on Pattern Analysis and Machine Intelligence, vol. 13, no.8, August, pp 834-841. 